\title{
Benefits of a New Annular Darkfield Method Using Köhler Illumination in a Energy Filtering TEM
}

\author{
Gerd Benner*, Erhard Zellmann, Stephan Hiller \\ *LEO Electron Microscopy Group, Carl Zeiss S-M-T AG, D-73446 Oberkochen, Germany
}

Darkfield illumination (DF) is a preferred method to visualize faint structures which are hardly seen in the brightfield (BF) mode. In DF the sample is illuminated by a tilted beam and the BF is blocked by an objective aperture. Thus, the image is formed by the scattered beam from the sample only. To provide a symmetrical (annular) DF two ways are possible: either a ring-shaped illumination aperture which provides an annular tilted beam on the specimen (Fig.3) is used, or the beam is conically rotated on the specimen (dynamical method). This paper describes a new DF which can be performed by a Köhler Illumination system (KIS) and has numerous advantages over the standard methods provided by conventional illumination systems.

In the KIS [1] the cross-over (emission pattern) is always imaged by the condenser lens system into the front focal plane of the final condenser lens (objective pre-field lens) which means the size (illumination angle), position (beam incidence), and the shape (conical DF) of this image define the illumination conditions. Instead of a persistent conically rotated cross-over image also the emission pattern of an under-heated $\mathrm{LaB}_{6}$ filament can be used to generate a ring shaped illumination in the front focal plane of the objective pre-field lens (see Fig.1a). As visualised in Fig 1, a small central spot is surrounded by an outer emission ring (a) for very low filament currents. Increasing the current the emission pattern moves from a large spot (b) to a small spot (c) known as "tip mode". If the current is furthermore increased four symmetrical off-axis emission areas appear (d) which collapse into a central spot (e) also known as "space charge mode". Fig.2 shows the ray-path of this DF mode. The KIS comprises a three-stage condenser lens zoom which images the emission pattern plane with variable (de)magnification into the front focal plane of the objective pre-field lens. With the central spot of the emission pattern (a) of an under-heated $\mathrm{LaB}_{6}$ filament blocked by a beam stop in the front focal plane, a ring shaped illumination can be achieved. In combination with an objective aperture then a DF image is obtained.

Compared to a conical illumination performed by deflectors, this illumination is free of dynamical artefacts and offers more brightness due to the simultaneous illumination from all angles. Compared to DF by a ring aperture this method has even more advantages: absolutely even illumination at all SA magnifications, which is not possible with a standard double condenser illumination system (Fig.3), where $\mathrm{C} 2$ has to be operated in cross over mode (focused) allowing DF only at high magnifications $(>30 \mathrm{kx})$. In addition with the new method the condenser zoom allows a wide range of tilt angles to obtain the optimum contrast from the sample. Variable and reproducible brightness conditions can be easily adjusted, permitting indulgent observation of beam sensitive specimens. Finally the emission pattern can be adjusted in a way that it fits into the objective aperture again to achieve BF illumination, too. By the built-in OMEGA Energy filter inelastic scattering from the sample is easily removed producing even crisper images with a better signal to noise ratio. As a proof of the excellent performance of this DF method Figs.4 and 5 show spread DNA strands [2] taken at different magnifications. Images have been acquired with a Proscan 1024x1024 pixel SSCCD camera with $5 \mathrm{sec}$ exposure on a LEO $912 \mathrm{AB}$ at $100 \mathrm{kV}$. All images are zero loss filtered with a $20 \mathrm{eV}$ Slit.

\section{References}

[1] G. Benner and W. Probst, Köhler Illumination in the TEM: fundamentals and advantages, Journal of Microscopy, 174-3 (1994), 133-142

[2] E. Le Cam, B. Theveny, B. Mignotte, B. Revet \& E. Delain, Quantitative electron microscopic analysis of DNA-protein interactions, J. Electron Micr. Tech., 18 (1991), 375-386 

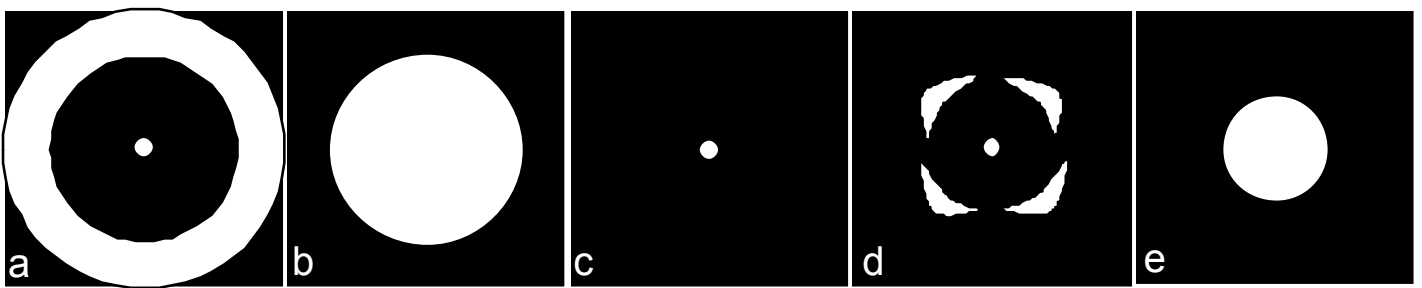

Fig.1: Emission pattern of $\mathrm{a} \mathrm{LaB}_{6}$ for different filament currents. For more explanations see text.

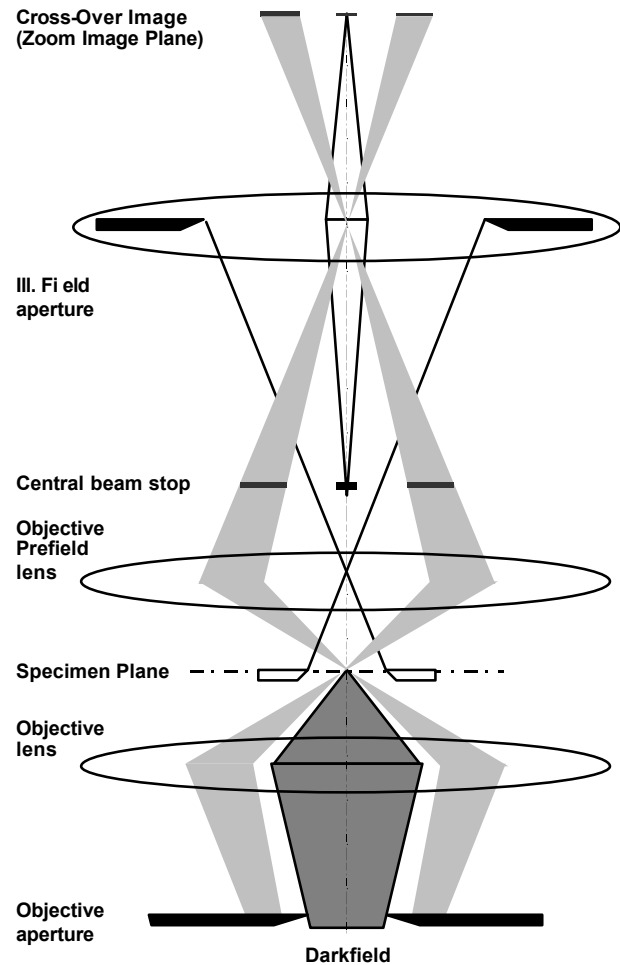

Fig.2: Darkfield with Koehler illumination
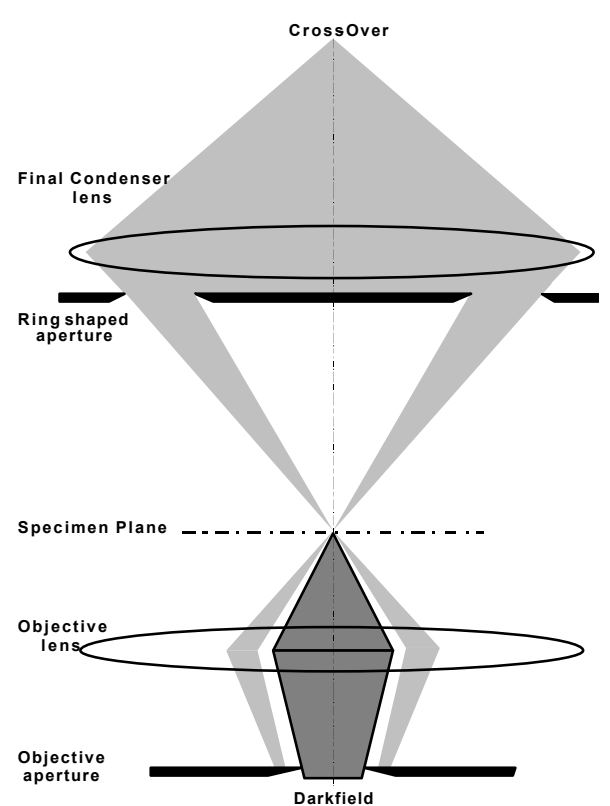

Fig.3: Conventional Darkfield

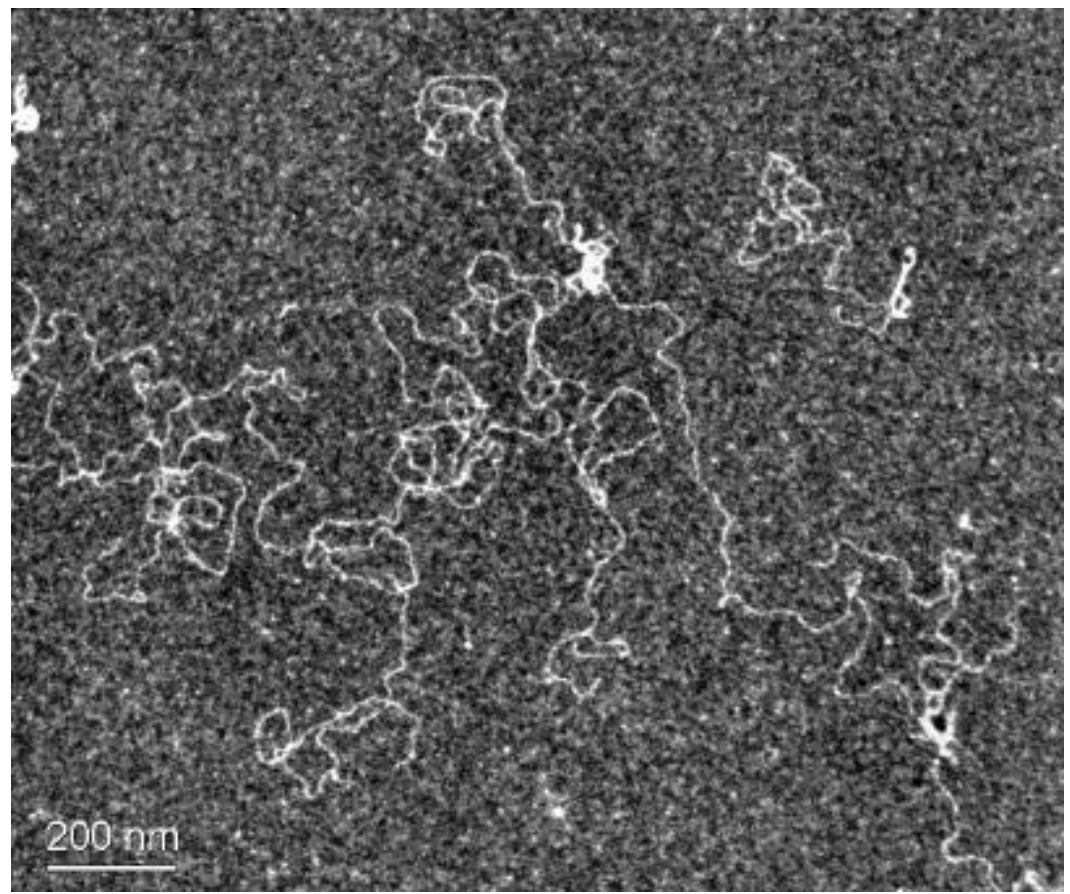

Fig.4: Zero loss filtered DNA strands at 10.000x mag *

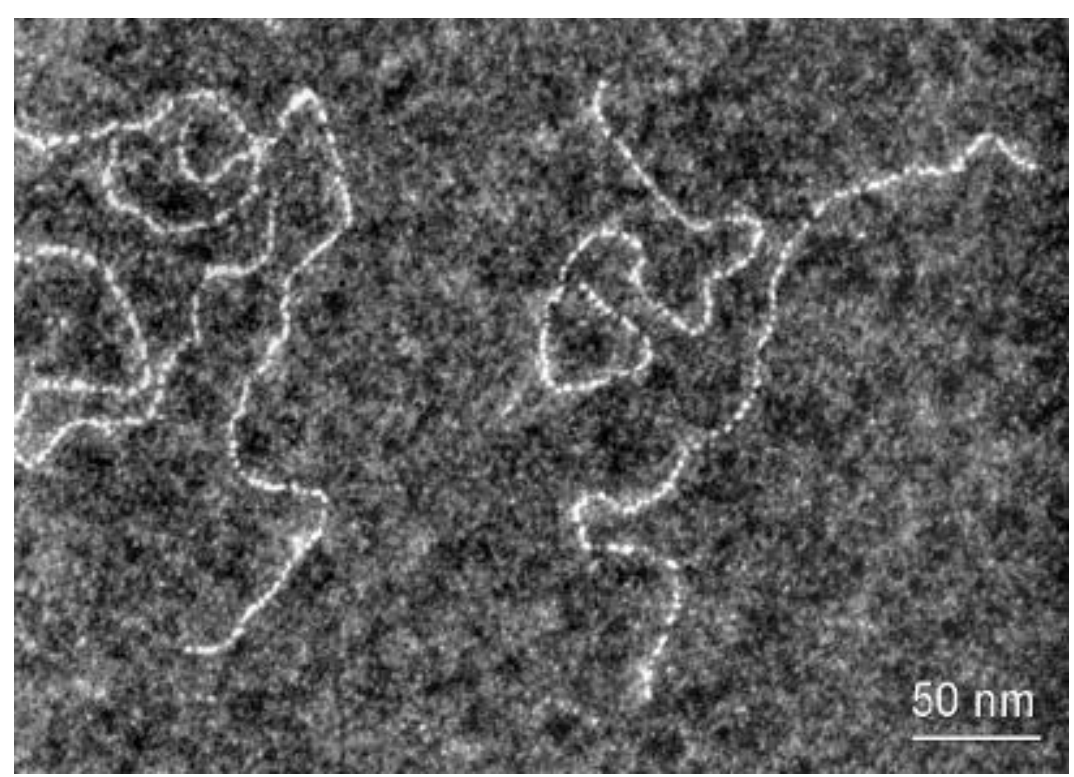

Fig.5: Zero loss filtered DNA strands at 40.000x mag*

* Specimen courtesy Dr. E. Delain, Institut Gustave-Roussy, 94805 Villjuif Cedex, France 